\title{
Hook Plate Fixation for Isolated Greater Tuberosity Fractures of the Humerus
}

\author{
Kyoung-Rak Lee, Ki-Cheor Bae, Chang-Jin Yon, Chul-Hyun Cho \\ Department of Orthopedic Surgery, Dongsan Medical Center, Keimyung University School of Medicine, Daegu, Korea
}

\begin{abstract}
Background: The purpose of this study was to investigate the outcomes after fixation using a 3.5-mm locking compression plate (LCP) hook plate for isolated greater tuberosity (GT) fractures of the proximal humerus.

Methods: We evaluated the postoperative radiological and clinical outcomes in nine patients who were followed up at least 1 year with isolated GT fractures. Using the deltopectoral approach, we fixed the displaced GT fragments with a 3.5-mm LCP hook plate (Synthes, West Chester, PA, USA). Depending on the fracture patterns, the hook plate was fixed with or without augmentation using either tension suture or suture anchor fixation.

Results: All the patient showed successful bone union. The mean time-to-union was 11 weeks. The radiological and clinical outcomes at the final follow-up were generally satisfactory. The mean visual analogue scale for pain, the University of California at Los Angeles score, the American Shoulder and Elbow Surgeons score, and the subjective shoulder value were 1.4, 30.3, 84.3, and 82.2\%, respectively. The mean active forward flexion, abduction, external rotation, and internal rotation of the shoulder were $156.7^{\circ}, 152.2^{\circ}, 61.1^{\circ}$, and the 10 th thoracic vertebral level, respectively. Only one patient presented with a postoperative complication of shoulder stiffness. The patient was treated through arthroscopic capsular release on the 5th postoperative month.

Conclusions: We conclude that fixation using 3.5-mm LCP hook plates for isolated GT fractures of the proximal humerus is a useful treatment method that provides satisfactory clinical and radiological outcomes.
\end{abstract}

(Clin Shoulder Elbow 2017;20(4):222-229)

Key Words: Hook plate fixation; Greater tuberosity fracture; Humerus

\section{Introduction}

Isolated greater tuberosity (GT) fractures of the proximal humerus are caused by high-energy trauma and often combined with a dislocation of the shoulder joint. Compared to other fractures of the proximal humerus, isolated GT fractures occur more commonly in younger patients than in older patients. ${ }^{1)}$ As the attachment site of the rotator cuff, the GT fragment of the proximal humerus is displaced posterosuperiorly because of the supraspinatus and infraspinatus tendons; this leads to a decrease in dynamic stability of the shoulder, causing limitations in abduction and external rotational movements and subacromial impingement. ${ }^{2,3)}$
The aims of surgical treatment of isolated GT fractures are to restore the normal anatomical position of the GT and to facilitate early motion exercise by achieving a rigid fixation. ${ }^{4)}$ Various fixation methods for GT fractures exist, including the transosseous suture fixation, the cannulated screw fixation, the plate fixation, the cerclage wire fixation, and the arthroscopic suture anchor fixation. ${ }^{3,5-11)}$ For patients with olecranon fractures or those who have undergone olecranon osteotomies, 3.5-mm locking compression plate (LCP) hook plate (Synthes, West Chester, PA, USA) is commonly used for fixation. For GT fixation, this plate has lower profile than the proximal humeral plates and, therefore, lower the chances of postoperative subacromial impingements. Moreover, strong fixation can be achieved by applying

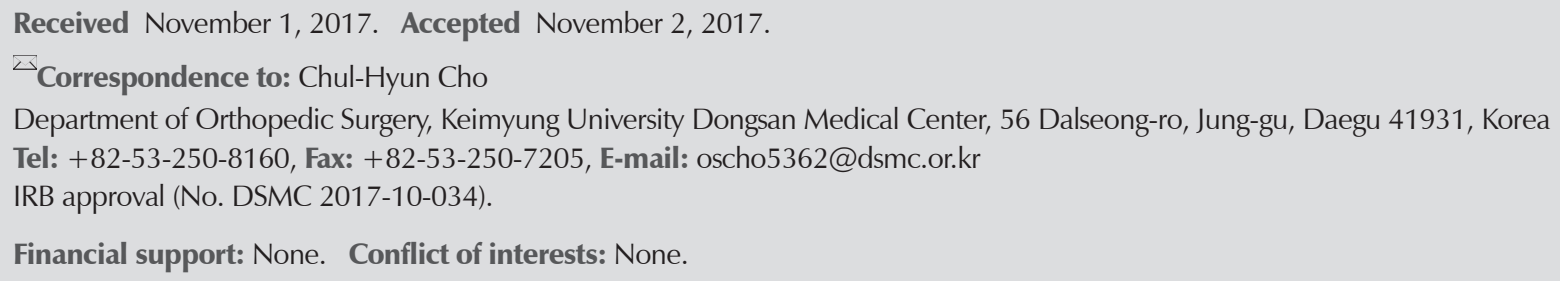


compression of the hook of the plate onto the fracture fragment of the GT. To obtain additional stability, augmentation tension suture with washers and cortical screws can be performed easily. Therefore, this study aimed to assess the clinical outcomes after 3.5-mm LCP hook plate fixation for isolated GT fractures of the proximal humerus.

\section{Methods}

\section{Subjects of Study}

This study has been exempted from informed consent of Institutional Review Board (IRB File No. DSMC 2017-10-034).

We enrolled nine patients who received plate fixation with 3.5 $\mathrm{mm}$ LCP hook plates for an isolated GT fracture of the proximal humerus from July 2011 to December 2015. We included only patients who could be followed-up for more than 12 months. The average age of the patients was 42.8 years (range, 17-76 years), and the average follow-up period was 37.8 months (range, 12-65 months). There were seven male and two female patients. The fracture was on the dominant arm in five patients. The cause of injury was in three patients each a simple fall, a motor vehicle accident, and an epileptic seizure. Six patients had a concomitant anterior dislocation of the shoulder, and five patients, a combined fracture at a different site. We performed the surgical treatment after an average of 6.9 days of the trauma (range, 1-18 days). Using images from plain radiography and computed tomography $(\mathrm{CT})$, we classified the fractures in accordance to the classification system developed by Mutch et al. ${ }^{12)}$ by shape-avulsion, split, or depression. We measured the distance of displacement on anteroposterior, external rotation, internal rotation, and axillary radiographs and selected the largest distance, and a displacement of $>5 \mathrm{~mm}$ was considered an indication for surgical treatment. The distance of displacement was measured after the reduction of the humeral head if a pa- tient had a combined shoulder dislocation (Table 1).

\section{Surgical Treatment}

The patient was administered general anesthesia and placed into supine position. Using the deltopectoral approach, we exposed the fracture site and pierced a No. 5 Ethibond (Ethicon, Somerville, NJ, USA) into the supraspinatus tendon, attached to the displaced GT fragment. We then pulled it in the anteroinferior direction to reduce the fracture fragment, and the reduction was confirmed with a C-arm. The reduction was maintained by keeping the suture tensioned, and the 3.5-mm LCP hook plate was positioned at the distal attachment site of the rotator cuff so that it holds the fracture fragments together. Again, the reduction and the plate position were confirmed with a C-arm. We performed temporary K-wire fixation for the maintenance of the reduction status. For comminuted or severely depressed fractures, compression and fixation of the GT fragment onto the inferior surface of the fracture site may cause the excessive inferior impaction of the GT fragment. This may induce excessive tension on the rotator cuff, fracture displacement even after operation. Therefore, in this situation, with maintaining adequate tension on the rotator cuff, we made a temporary fixation of the plate so that the medial surface of the fracture fragment was compressed. We positioned the plate against the proximal humerus by inserting cortical screws through the 2 nd or 3rd screw holes initially and then into the remaining screw holes for the final fixation. The $\mathrm{C}$-arm was used to assess the reduction and the screw length. When we performed augmentation tension sutures, we inserted a cortical screw that was $2 \mathrm{~mm}$ longer than the distance measured to the distal cortex into the 4th screw hole. We threaded two differently sized washers onto the cortical screw (3.5-mm and 4.5-mm washers) and left it unfastened. We then fastened the cortical screw after inserting two or three No. 5 Ethibond sutures through the rotator cuff and the between

Table 1. The Demographic Data of Patients

\begin{tabular}{|c|c|c|c|c|c|c|c|c|c|}
\hline $\begin{array}{l}\text { Patient } \\
\text { No. }\end{array}$ & $\begin{array}{l}\text { Age } \\
(\mathrm{yr})\end{array}$ & Sex & $\begin{array}{l}\text { Affected } \\
\text { side }\end{array}$ & $\begin{array}{c}\text { Injury } \\
\text { mechanism }\end{array}$ & Dislocation & Classification & Combined injury & $\begin{array}{c}\text { Time to } \\
\text { surgery (d) }\end{array}$ & $\begin{array}{l}\text { Follow-up } \\
\text { (mo) }\end{array}$ \\
\hline 1 & 76 & M & Rt. & Fall down & + & A \& S & Rt. acetabular Fx., MRF, ICH & 11 & 65 \\
\hline 2 & 25 & M & Lt. & MVA & & A & Lt. clavicle Fx., MRF & 3 & 59 \\
\hline 3 & 30 & M & Lt. & Fall down & + & A & - & 1 & 54 \\
\hline 4 & 62 & $\mathrm{~F}$ & Rt. & MVA & + & A & Rt. tibia plateau Fx., pelvic bone Fx. & 6 & 38 \\
\hline 5 & 17 & M & Lt. & Seizure & + & $\mathrm{D}$ & - & 4 & 31 \\
\hline 6 & 51 & M & Rt. & MVA & & A & Rt. distal femur open Fx., pelvis bone Fx. & 18 & 29 \\
\hline 7 & 58 & $\mathrm{~F}$ & Rt. & Seizure & + & S \& D & Lt. proximal humerus Fx. & 10 & 28 \\
\hline 8 & 21 & M & Rt. & Seizure & + & $\mathrm{D}$ & - & 6 & 24 \\
\hline 9 & 45 & M & Lt. & Fall down & & A & - & 2 & 12 \\
\hline
\end{tabular}

M: male, F: female, Rt.: right, Lt.: left, MVA: motor vehicle accident, A: avulsion, S: split, D: depression, Fx.: fracture, MRF: multiple rip fracture, ICH: intracerebral hemorrhage. 
two washers and tightened it, inducing greater tension on the suture on the washers. For severely comminuted GT fractures, we inserted a suture anchor on the proximal fracture site before plating and passed the suture through the rotator cuff, thereby, reducing the GT fracture. Then the hook plate was positioned as above (Fig. 1). Postoperatively, an abduction brace was applied for 6 weeks. Passive and active range of motions (ROMs) were permitted from the 2 nd and 6th week of treatment, respectively.

\section{Assessment of Outcomes}

We assessed the following radiological parameters during follow-up: bone union and time-to-union. The time-to-union was defined as the point when cortical continuity was observed on one of three planes of plain radiography (anteroposterior, external rotation, or internal rotation) and when tenderness at the fracture site resolved. ${ }^{6}$ We used the method employed by Gillespie et al. ${ }^{8}$ to evaluate the postoperative anatomical reduction and the extent of displacement, if any. We defined an anatomical reduction as a distance between the GT and the humeral head of $4-10 \mathrm{~mm}$ at the final follow-up. Similarly, we defined a loss in reduction as a displacement of greater than $3 \mathrm{~mm}$ at the final follow-up compared to the immediate postoperative radiograph. We evaluated clinical outcomes in terms of the level of pain and functional restoration of daily activities. The following parameters of clinical outcome were evaluated at the final follow-up: visual analogue scale (VAS) for pain, the University of California at Los Angeles (UCLA) score, the American Shoulder and Elbow Surgeons (ASES) score, the subjective shoulder value (SSV), and the ROM. Postoperative complications were also evaluated.

\section{Results}

We observed bone union in all the patients. The average time-to-union was 11 weeks (range, 7-12 weeks), and the average distance between the humeral head and the GT was 9.7 mm (range, 5-25 mm). Although two patients (patient No. 1 and 2) did not achieve anatomical reduction, they showed bone
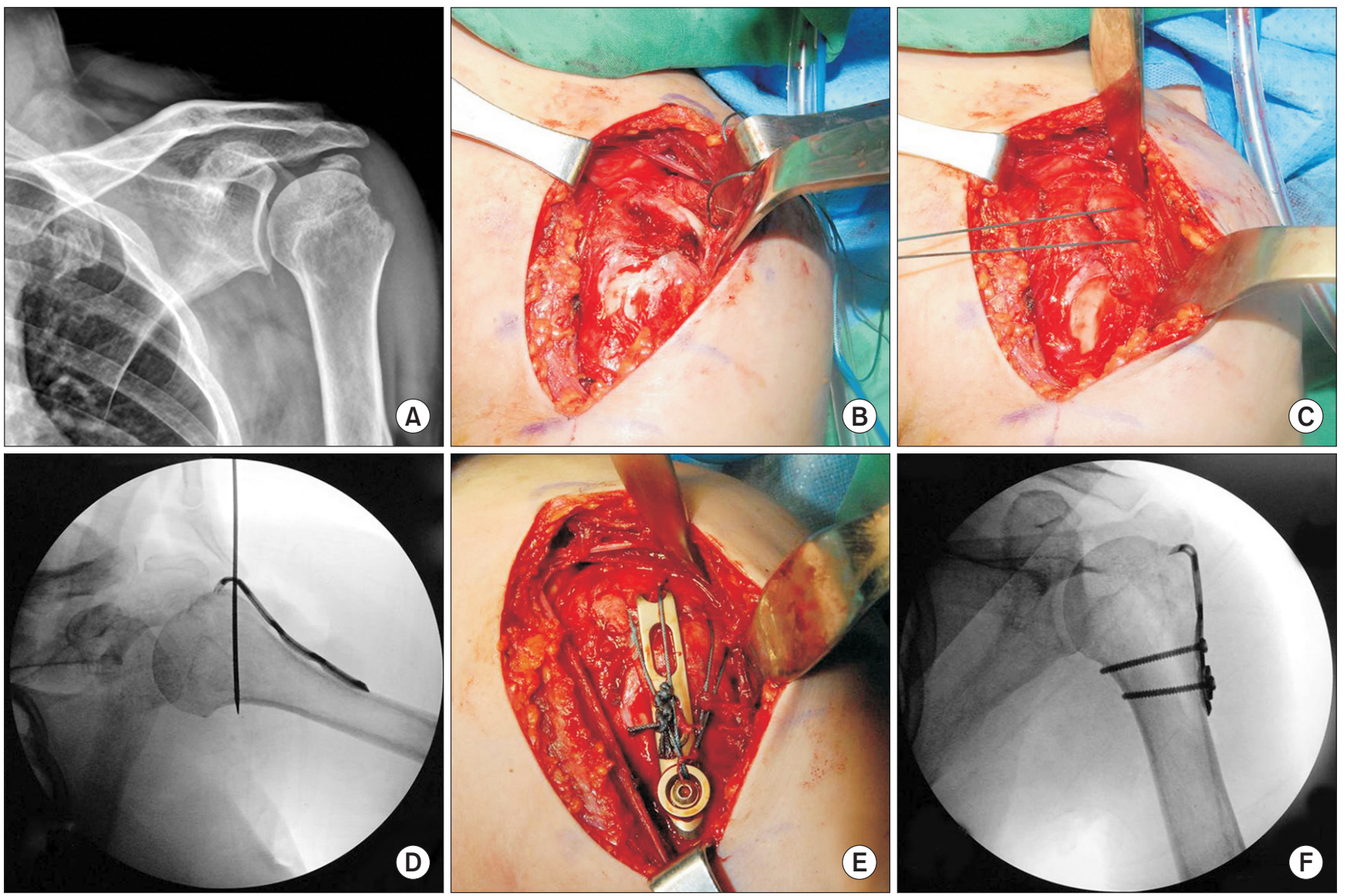

Fig. 1. (A) An avulsion-type isolated fracture of the greater tuberosity (GT) of the left proximal humerus on a simple anteroposterior radiograph. (B) The rotator cuff tendon is tagged with a No. 5 Ethibond suture for the reduction of the GT fracture fragment. (C) The tagged rotator cuff tendon is pulled in the anteroinferior direction to reduce the GT fracture fragment. (D) After attachment of the 3.5-mm locking compression hook plate, a temporary K-wire is threaded through most of the proximal screw holes to maintain the reduction of the GT fragment and the plate. (E, F) Augmentation tension-band suture fixation is performed using two washers. 
union, favorable clinical outcomes, and no additional displacement. None of the patients showed more than $3 \mathrm{~mm}$ of fracture displacement postoperatively. We observed the following average clinical scores at the final follow-up: a VAS for pain of 1.4 (range, 0-5); a UCLA score of 30.3 (range, 24-35); an ASES score of 84.3 (range, 58-100); and an SSV score of $82.2 \%$ (range, $50 \%-100 \%)$. At the final follow-up, the average active ROMs were $156.7^{\circ}$ for forward flexion (range, $150^{\circ}-170^{\circ}$ ), $152.2^{\circ}$ for abduction (range, $130^{\circ}-160^{\circ}$ ), $61.1^{\circ}$ external rotation (range, $60^{\circ}-70^{\circ}$ ), and the 10th thoracic vertebra for internal rotation (range, 4th lumbar vertebra-7th thoracic vertebra) (Table 2).

One patient presented with a postoperative complication of shoulder stiffness. On the 6th postoperative week, the passive ROMs of the affected shoulder were $90^{\circ}$ of forward flexion, $100^{\circ}$ of abduction, and $20^{\circ}$ of external rotation, showing a clear restriction in ROM in comparison to the contralateral arm. We administered an intraarticular injection of steroids and aggressive rehabilitation from the 3 rd postoperative month. Because no improvement was observed, we removed the plate and performed arthroscopic capsular release on the 5th postoperative month. Other complications such as fixation loss, infection, and neural or vascular injuries were not seen.

\section{Case 1}

A 51-year-old male patient (patient No. 6) was hospitalized for unstable vital signs and multiple fractures after a car accident. An isolated GT fracture of the right proximal humerus was diagnosed. On the 18th day of the accident, we performed two types of fixations: plate fixation using the 3.5-mm LCP hook plate and tension sutures, for which two washers were used. We observed bone union on the 12th week of treatment and satisfactory clinical outcomes at the final follow-up (Fig. 2).

\section{Case 2}

A 17-year-old male patient (patient No. 5), who had an underlying disease of epilepsy, was hospitalized after an epileptic seizure due to irregular use of anti-epileptic drugs. The seizure caused a combination of an anterior dislocation of the shoulder and a GT fracture of the left proximal humerus. We performed a closed reduction of the dislocation immediately and plate fixation using a 3.5-mm LCP hook plate on the 4th day after injury. We observed bone union on the 7th postoperative week and excellent clinical outcomes at the final follow-up (Fig. 3).

\section{Discussion}

In this study, we found that fixation using a 3.5-mm LCP hook plate, for isolated GT fractures of the humerus provides satisfactory radiological and clinical outcomes. Moreover, we found that the stable fixation achieved through the hook helps avoid common postoperative complications such as re-displacement of the GT.

The extent of displacement of the fracture fragment necessary to give an indication of surgical treatment for isolated GT fractures is still under debate. Traditionally, a displacement of $>$ $1 \mathrm{~cm}$, or $>3 \mathrm{~mm}$ if the patient is young and active or engaged in overhead activities, has been used as the indication for surgical treatment. ${ }^{2)}$ However, many authors agree that a displacement of $>5 \mathrm{~mm}$ is likely to cause clinically significant symptoms such as shoulder impingement. ${ }^{2,13,14)}$ In fact, $50 \%$ to $60 \%$ of fractures with a displacement of $<5 \mathrm{~mm}$ show exacerbated displacement after conservative treatment, especially in younger patients. ${ }^{15,16)}$ Our study suggest that a displacement of $>5 \mathrm{~mm}$ measured on plain radiography or $\mathrm{CT}$ should be an indication for surgical treatment. Therefore, it is important to make an accurate diagnosis and treatment decisions that take into account not only

Table 2. The Results of Surgical Treatment Using $3.5 \mathrm{~mm}$ LCP Hook Plate for Isolated Humeral GT Fracture

\begin{tabular}{|c|c|c|c|c|c|c|c|c|c|c|c|c|}
\hline \multirow{2}{*}{ Patient } & \multirow{2}{*}{$\begin{array}{c}\text { Union } \\
\text { time (wk) }\end{array}$} & \multirow{2}{*}{$\begin{array}{c}\text { GT-head } \\
\text { height }(\mathrm{mm})\end{array}$} & \multirow{2}{*}{ Displacement } & \multirow{2}{*}{$\begin{array}{l}\text { UCLA } \\
\text { score }\end{array}$} & \multirow{2}{*}{$\begin{array}{l}\text { ASES } \\
\text { score }\end{array}$} & \multirow{2}{*}{ SSV } & \multirow{2}{*}{ VAS } & \multicolumn{4}{|c|}{ ROM } & \multirow{2}{*}{ Complication } \\
\hline & & & & & & & & $\mathrm{FF}\left({ }^{\circ}\right)$ & $\operatorname{Abd}\left({ }^{\circ}\right)$ & $\operatorname{ER}\left({ }^{\circ}\right)$ & IR & \\
\hline 1 & 12 & 65 & - & 33 & 80 & 70 & 2 & 150 & 150 & 60 & L4 & - \\
\hline 2 & 11 & 59 & - & 35 & 95 & 95 & 1 & 160 & 160 & 60 & T7 & - \\
\hline 3 & 12 & 54 & - & 35 & 93 & 95 & 1 & 160 & 160 & 60 & $\mathrm{~T} 7$ & - \\
\hline 4 & 10 & 38 & - & 27 & 58 & 50 & 5 & 150 & 150 & 60 & $\mathrm{~T} 7$ & Stiffness \\
\hline 5 & 7 & 31 & - & 29 & 95 & 90 & 0 & 170 & 160 & 70 & T10 & - \\
\hline 6 & 12 & 29 & - & 30 & 70 & 80 & 2 & 150 & 150 & 60 & T11 & - \\
\hline 7 & 12 & 28 & - & 30 & 88 & 80 & 0 & 150 & 130 & 60 & L4 & - \\
\hline 8 & 12 & 24 & - & 30 & 100 & 100 & 0 & 160 & 160 & 60 & T7 & - \\
\hline 9 & 11 & 12 & - & 24 & 78 & 80 & 2 & 160 & 150 & 60 & T10 & - \\
\hline
\end{tabular}

LCP: locking compression plate, GT: greater tuberosity, UCLA: University of California at Los Angeles, ASES: American Shoulder and Elbow Surgeons, SSV: subjective shoulder value, VAS: visual analogue scale, ROM: range of motions, FF: forward flexion, Abd: abduction, ER: external rotation, IR: internal rotation. 

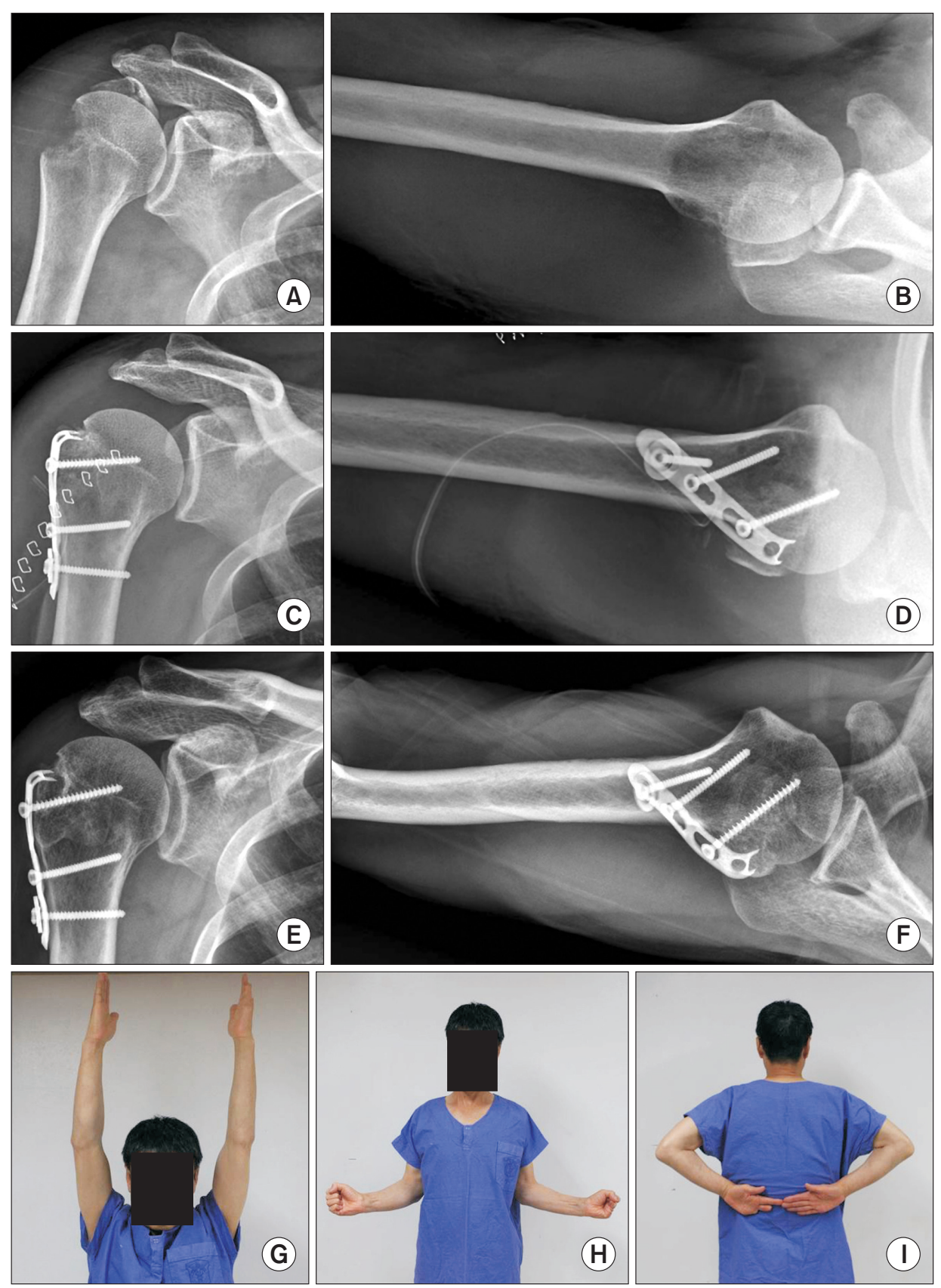

Fig. 2. (A, B) Initial anteroposterior (AP) and axillary radiographs of a 51-year-old male patient show an avulsion-type isolated GT fracture of the proximal humerus on the right shoulder. (C, D) Immediate postoperative AP and axillary radiographs of $3.5-\mathrm{mm}$ locking compression hook plate fixation with augmentation by tension suture fixation. (E, F) Postoperative 1-year AP and axillary radiographs show complete union of the fracture. (G-I) At the final follow-up, the patient had a satisfactory clinical outcome and an active range of motion. patient characteristics and fracture type but also surgeon preference and expertise for displaced GT fractures.

The methods of numerous surgical treatments, along with their limitations, of isolated GT fractures of the humerus have been described. For instance, fixation using cannulated screws alone or in combination with washers through a small incision window or subcutanaeous fixation using K-wires are commonly used in patients with large fracture fragments of good bone quality. However, it is difficult to provide stable fixation because of poor bone quality in patients with osteoporosis. ${ }^{6,10,17)}$ In contrast, previous studies have shown that trans-osseous repairs using non-absorbable sutures provide rigid fixation even in patients with osteoporosis or with comminuted fractures. However, for large fracture fragments it achieves relatively weak fixation, leading to a delayed rehabilitation among patients with large fracture fragments. ${ }^{6,10,17)}$ Plate fixation has been shown to provide rigid fixation in even large fracture fragments and be biomechanically superior than non-absorbable suture or screw fixation. ${ }^{3,9,18)}$ Plate fixation may be sufficient for split fractures with relatively large fracture fragments because many screws can be inserted; however, it may not be sufficient for avulsed or depressed fractures, fractures with small fragments, or those combined with comminution. ${ }^{4,18)}$ To complement this, augmentation technique using suture fixation, cerclage K-wire fixation, or suture anchor fixation 

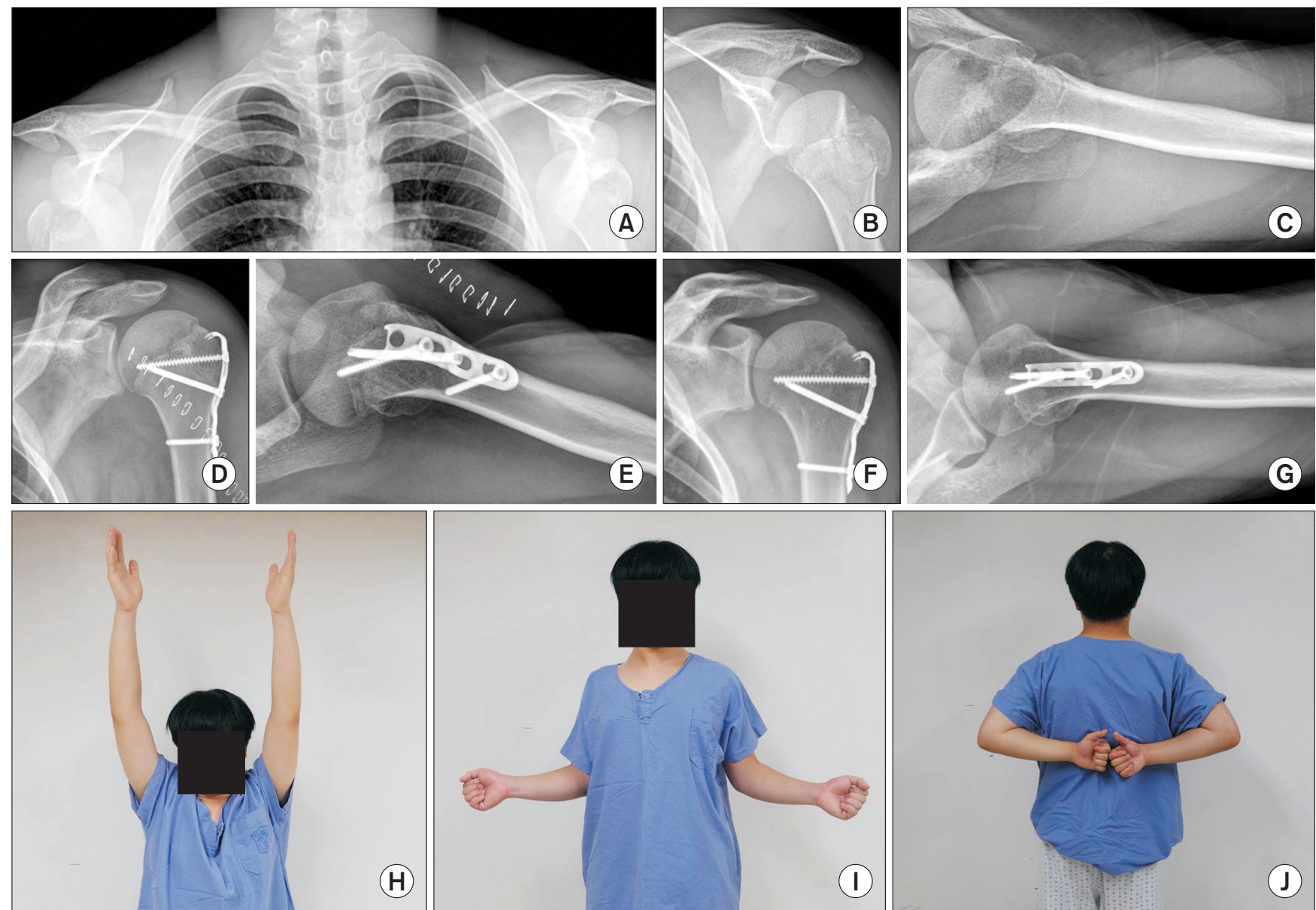

(H)
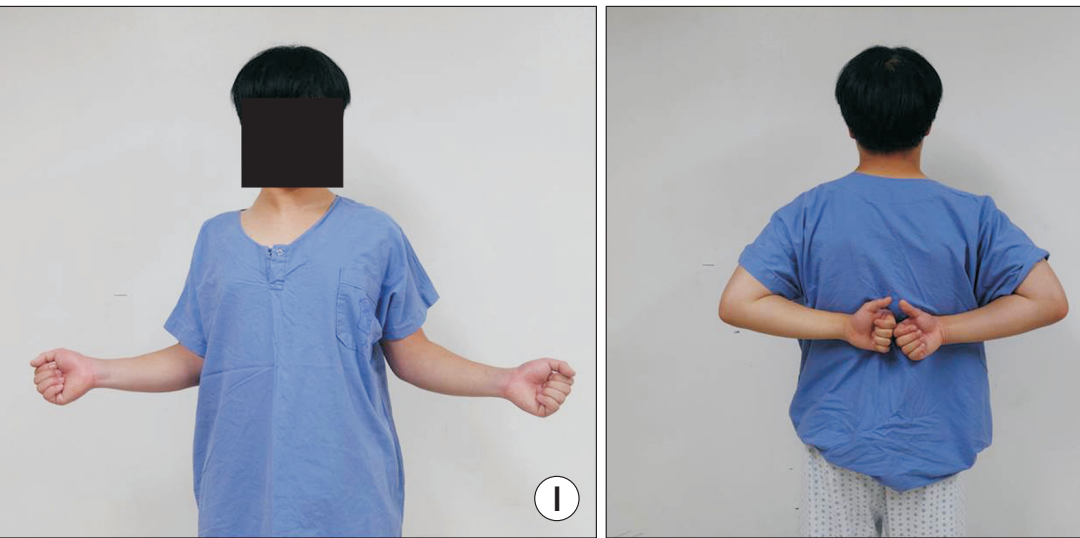

Fig. 3. (A) An initial radiograph of the proximal humerus in a 17-year-old male patient shows a bilateral and anteriorly displaced greater tuberosity (GT) fracture. (B, C) The anteroposterior (AP) and axillary radiographs after closed reduction show an avulsion-type isolated GT fracture of the left proximal humerus. (D, E) Immediate postoperative AP and axillary radiographs show an anatomically reduced fracture stabilized with a 3.5-mm locking compression hook plate. (F, G) The 12-month follow-up AP and axillary radiographs show complete union of the fracture. (H-J) At the final follow-up, the patient had a satisfactory clinical outcome and an active range of motion.

are sometimes can be applied. ${ }^{4,7)}$

One of the advantages of the 3.5-mm LCP hook plates used in this study is that they can stabilize fragments of even small and comminuted fractures and, therefore, prevent loss in reduction. ${ }^{19)}$ Other advantages include the buttressing effect of the plate, which supports the fracture. Moreover, compared to preexisting LCP plates used in proximal humeral fractures, these plates are lower in profile so they are less likely to induce subacromial impingement. Shin et al. ${ }^{10)}$ described a modified spring plate that was made of $1 / 3$ tubular plate being cut. The elasticity of end point of plate help to fix the GT fractures. Their novel treatment method was found to be associated with favorable clinical outcomes. Nevertheless, 3.5-mm LCP plates could be superior to spring plates because it can stabilize fractures through the hook and provide rigid fixation through locking screws especially for osteoporotic fractures. Cho et al. ${ }^{20,21)}$ performed augmentation tension suture fixation of the rotator cuff to prevent varus deformity and displacement of the GT fracture after proxi- mal humerus internal locking system (PHILOS) plate (Synthes, West Chester, PA, USA) fixation for proximal humerus fractures. They suggested it could be an effective treatment method by using two differently sized washers $(3.5 \mathrm{~mm}$ and $4.5 \mathrm{~mm}$ ) to perform a tension suture and obtained satisfactory outcomes. ${ }^{20}$ Using the same suture-tensioning method, we performed a concomitant 3.5-mm LCP hook plate fixation for isolated GT fractures and an augmentation tension suture fixation of the rotator cuff, using cortical screws and two differently sized washers. For severely comminuted fractures, we applied the trapdoor technique using suture anchor developed by McLaughlin-Symon et al. ${ }^{11)}$ by replacing the sutureless anchor with a 3.5-mm LCP hook plate.

In this study, all patients achieved a successful bone union at an average 11 weeks of treatment without fracture re-displacement or fixation loss. We found that the treatment led to satisfactory clinical outcomes in most patients. However, anatomical reduction was not achieved in two patients; one patient had a 
severe comminution fracture and the other did not have severe comminution but had a small fracture fragment. Although both patients had an depression, which was inferior to the anatomical position, the clinical outcomes were not so bad. A small degree of depression is not thought to have much influence on clinical outcome because the rotator cuff maintains optimal tension and that there is bone union without posterosuperior displacement. A patient who showed poor outcomes in all parameters of clinical outcome had had postoperative shoulder stiffness and received an arthroscopic capsular release on the 5th month of treatment. At the final follow-up, this patient showed a satisfactory ROM but recalcitrant pain and restriction in daily activities. The patient did not have any underlying or co-morbid diseases. The patient's postoperative shoulder stiffness may be attributable to several reasons. The patient's shoulder was dislocated anteroinferiorly for at least 4 hours until the patient had arrived at the emergency room. The post-reduction clinical assessment of the patient's partial brachial plexus injury was conducted when the patient still had abnormal sensations of the forearm and hand, numbness, and restricted finger movement. Moreover, the patient had multiple traumatic injuries, a combined pelvic bone fracture and ipsilateral tibial plateau fracture, which were treated conservatively and surgically, respectively. Therefore, patient compliance to rehabilitation exercises may have been low.

Limitations of this study include a small study population and a retrospective uncontrolled study design. In the future, studies with a prospective study design are required to compare the clinical and radiological outcomes and the biomechanical analysis between 3.5-cm LCP hook plate fixation and another mode of fixation for GT fractures.

\section{Conclusion}

We found that fixation using 3.5-mm LCP hook plate for isolated GT fractures of the proximal humerus leads to satisfactory clinical and radiological outcomes and it may be a useful surgical technique for firm fracture fixation.

\section{References}

1. Green A, Izzi J Jr. Isolated fractures of the greater tuberosity of the proximal humerus. J Shoulder Elbow Surg. 2003;12(6):641-9.

2. Gruson Kl, Ruchelsman DE, Tejwani NC. Isolated tuberosity fractures of the proximal humeral: current concepts. Injury. 2008;39(3):284-98.

3. Chen YF, Zhang W, Chen Q, Wei HF, Wang L, Zhang CQ. AO X-shaped midfoot locking plate to treat displaced isolated greater tuberosity fractures. Orthopedics. 2013;36(8):e995-9.

4. Park SE, Jeong JJ, Panchal K, Lee JY, Min HK, Ji JH. Arthroscopic-assisted plate fixation for displaced large-sized comminuted greater tuberosity fractures of proximal humerus: a novel surgical technique. Knee Surg Sports Traumatol Arthrosc. 2016;24(12):3892-8.

5. Brais G, Ménard J, Mutch J, Laflamme GY, Petit Y, Rouleau DM. Transosseous braided-tape and double-row fixations are better than tension band for avulsion-type greater tuberosity fractures. Injury. 2015;46(6):1007-12.

6. Cho NS, Moon SC, Rhee YG. Clinical results of various surgical techniques for isolated fracture of greater tuberosity of humerus. J Korean Fract Soc. 2013;26(2):133-9.

7. Bockmann B, Buecking B, Eschbach DA, Franz D, Ruchholtz S, Mohr J. Fixation of the greater tuberosity in proximal humeral fractures: FiberWire ${ }^{\circledR}$ or wire cerclage? Acta Orthop Belg. 2015;81(1):9-16.

8. Gillespie RJ, Johnston PS, Gordon VA, Ward PJ, Getz CL. Using plate osteosynthesis to treat isolated greater tuberosity fractures. Am J Orthop (Belle Mead NJ). 2015;44(8):E248-51.

9. Schöffl V, Popp D, Strecker W. A simple and effective implant for displaced fractures of the greater tuberosity: the "Bamberg" plate. Arch Orthop Trauma Surg. 2011;131(4):509-12.

10. Shin DJ, Byun YS, Chang SA, Yun HM, Park HW, Park JY. The surgical outcomes of isolated greater tuberosity fractures of the proximal humerus fixed with the spring plate. J Korean Fract Soc. 2009;22(3):159-65.

11. McLaughlin-Symon I, Kenyon P, Morgan B, Ravenscroft M. A new "trapdoor technique" for fixation of displaced greater tuberosity fractures of the shoulder. J Hand Microsurg. 2015;7(2):241-3.

12. Mutch J, Laflamme GY, Hagemeister N, Cikes A, Rouleau DM. A new morphological classification for greater tuberosity fractures of the proximal humerus: validation and clinical implications. Bone Joint J. 2014;96(5):646-51.

13. Neer CS 2nd. Displaced proximal humeral fractures. Part I. Classification and evaluation. By Charles S. Neer, I, 1970. Clin Orthop Relat Res. 1987;(223):3-10.

14. Park TS, Choi IY, Kim YH, Park MR, Shon JH, Kim Sl. A new suggestion for the treatment of minimally displaced fractures of the greater tuberosity of the proximal humerus. Bull Hosp Jt Dis. 1997; 56(3):171-6.

15. Platzer P, Kutscha-Lissberg F, Lehr S, Vecsei V, Gaebler C. The influence of displacement on shoulder function in patients with minimally displaced fractures of the greater tuberosity. Injury. 2005;36(10):1185-9.

16. Hébert-Davies J, Mutch J, Rouleau D, Laflamme GY. Delayed migration of greater tuberosity fractures associated with anterior shoulder dislocation. J Orthop Trauma. 2015;29(10):e396400.

17. Moon ES, Kim MS, Kim YJ. The surgical outcomes for isolated greater tuberosity fracture of proximal humerus. J Korean Fract Soc. 2007;20(3):239-45.

18. Gaudelli C, Ménard J, Mutch J, Laflamme GY, Petit Y, Rouleau 
DM. Locking plate fixation provides superior fixation of humerus split type greater tuberosity fractures than tension bands and double row suture bridges. Clin Biomech (Bristol, Avon). 2014;29(9):1003-8.

19. Lee SK, Park JS, Choy WS. Locking compression plate distal ulna hook plate as alternative fixation for fifth metatarsal base fracture. J Foot Ankle Surg. 2014;53(5):522-8.
20. Cho $\mathrm{CH}$, Jung $\mathrm{GH}$, Song KS. Tension suture fixation using 2 washers for proximal humeral fractures. Orthopedics. 2012;35(3):202-5.

21. Cho $\mathrm{CH}$, Kim DH, Kim BS. Radiographic and clinical results of tension suture fixation using two washers with PHILOS plate for proximal humeral fractures. Injury. 2017;48(2):464-8. 